\title{
Assembling of Magnetic Iron Oxide Nanoparticles Controlled by Self- Assembled Monolayers of Functional Coordinating or Chelating Trialkoxysilanes
}

\author{
Benoit P. Pichon ${ }^{*}$, , Paulin Buchwalter ${ }^{1}$, Carole Carcel$^{2}$, Xavier Cattoën ${ }^{2}$, Michel Wong Chi Man² \\ and Sylvie Begin-Colin ${ }^{1}$
}

\author{
${ }^{I}$ Institut de Physique et de Chimie des Matériaux de Strasbourg, UMR 7504, CNRS-UdS-ECPM, 23 rue du Loess, BP \\ 43, F-67034 Strasbourg Cedex 2, France \\ ${ }^{2}$ Institut Charles Gerhardt de Montpellier, UMR 5253 CNRS-UM2-ENSCM-UM1, 8, rue de l'école normale, 34296 \\ Montpellier, France
}

\begin{abstract}
Magnetic nanoparticles (NPs) have been assembled in films by using self-assembled monolayers (SAMs) of organic molecules. SAMs were prepared by the reaction of organotrialkoxysilanes with surface silanol groups of silicon wafers. SAMs were prepared and their surface was decorated either by amine or thymine groups which have the ability to immobilize iron oxide nanoparticles as monolayers. These groups have different coordinating and chelating abilities and influence the kinetic of the assembling reaction. Finally, the magnetic properties of the assemblies of NPs were studied.
\end{abstract}

Keywords: Iron oxide nanoparticle, self-assembled monolayer, magnetism, assembly, organotrialkoxysilane.

\section{INTRODUCTION}

The assembling of magnetic nanoparticles (NPs) into arrays represents a very exciting and important challenge regarding their high potentials in the development of new nanodevices for spintronic, magnetic and magneto-electronic applications [1-3]. It is well argued that the key to successful applications of such nanoparticle-based devices is the engineering of the assembling of nanoparticles. Therefore, assembling methods are developed to organize nanoparticles on surfaces.

Most films made from nanoparticles which consist in 2D and 3D arrays are generally obtained by "bottom-up" approaches based on self-assembly [4]. Such a method is very well suited to the use of non aggregated inorganic nanoparticles which are considered as building blocks to spontaneously assemble into arrays on substrates. Although the synthesis of magnetic nanoparticles with high control over their physicochemical properties such as size, shape and crystallinity is well reported, their assembling with high control still remains a great challenge. The main difficulties arise from the control of the film formation over macroscopic distances which is mandatory for enabling meaningful studies of the film properties. Among the various techniques enabling the deposition of nanoparticles on large areas, the approach using chemically well-addressed surfaces such as self-assembled monolayers (SAMs) of organic molecules consist in addressing the assembling of NPs. SAMs represent a versatile approach to functionalize surfaces with specific functional groups which have the

*Address correspondence to this author at the Institut de Physique et de Chimie des Matériaux de Strasbourg, UMR 7504, CNRS-UdS-ECPM, 23 rue du Loess, BP 43, F-67034 Strasbourg Cedex 2, France; Tel: + 33 (0) 3 881071 33; Fax: + 33 (0) 3881072 47; E-mail: Benoit.Pichon@unistra.fr ability to immobilize NPs. Only few studies report the use of SAMs to prepare arrays of magnetic NPs. Bio-functionalized manganese ferrite were specifically assembled onto a SAM on the basis of molecular recognition [5]. On the other hand the direct immobilization of $\mathrm{PbSe}, \mathrm{PbS}$, $\mathrm{Co}$ or $\mathrm{Fe}_{3} \mathrm{O}_{4} \mathrm{NPs}$ onto SAMs lead to non homogeneous films where the density in NPs and the orientation of molecules of the SAM influence the magnetic microstructure of the assemblies [69]. Very recently, we reported on the chemical assisted assembling of iron oxide NPs controlled by specific functional head groups at the SAM surface [10]. The deposition of NPs was demonstrated to strongly depend on the chelating and coordinating abilities of these terminal groups. Indeed, SAMs displaying carboxylate head groups with high chelating abilities were found to anchor rapidly NPs with a high density. Hydroxide groups which are better coordinating groups also induced the deposition of NPs but the kinetic was slower. Finally, no deposition of NPs was observed with non-interacting methyl head groups showing the importance of the former coordinating or chelating functional groups with the NPs. Mixed SAMs which display carboxylic and methylene groups with different molar ratios were accurate to pattern the substrate and thus to obtain patterned deposition of NPs [11-13]. Large assemblies of tight packed NPs or cluster of few NPs were formed selectively. It resulted in the control of the magnetic properties of the film as a function of the domain size which directs the strength of dipolar interactions between NPs. Therefore the nature of terminal groups at the SAMs surface has a strong influence on the deposition of NPs on large areas.

Here, we report on the assembling of magnetic iron oxide nanoparticles controlled by SAMs of alkoxysilanes-based molecules. The self-assembling of such molecules was performed by the covalent bonding at the surface of a silicon 
wafer which results in much more stable systems than SAMs prepared by the adsorption of thiol molecules on gold surfaces which is reversible [12]. Such approach to prepare SAMs is also very interesting because of the very low roughness of silicon wafers compared with that of gold surfaces [13]. Two different kinds of SAMs have been prepared to decorate the silicon wafer surface with amine and thymine terminal groups which are both polar but have different abilities to immobilize nanoparticles and should control the assembling of NPs.

\section{EXPERIMENTS}

Chemicals and materials. 3-(aminopropyl)triethoxysilane (APTES) was purchased from Aldrich. The N-1(Triethoxysilylundecyl)thymine (THY) was synthesized according to our previous report [14]. Tetrahydrofurane (THF) was used as received. Toluene was distilled and dried on sodium. The silicon wafers were cleaned and activated by using a plasma generated from a mixture of oxygen and argon for 2 minutes.

Preparation of iron oxide nanoparticles (NPs). $12 \mathrm{~nm}$ sized iron oxide NPs which crystallize in the spinel structure were produced by the thermal decomposition method [15] and following the exact procedure we reported previously $[16,17]$. The preparation of an iron(III)/oleate complex ( $\left.\mathrm{Fe}(\text { oleate })_{3}\right)$ which is thermally decomposed in a high boiling solvent in the presence of oleic acid was performed following the exact procedure we reported previously [22]. $\mathrm{Fe}(\text { oleate })_{3}$ was prepared from $\mathrm{FeCl}_{3} \cdot \mathrm{H}_{2} \mathrm{O}$ (10.8 g, $40 \mathrm{mmol}$, $97 \%$, Aldrich) which was dissolved in $60 \mathrm{ml} \mathrm{H}_{2} \mathrm{O}$ (Milli-Q) and $80 \mathrm{~mL}$ ethanol. This solution was mixed with a solution of sodium oleate (36.5 g, $120 \mathrm{mmol}, 82 \%$, Riedel-de Haën) dissolved in hexane $(140 \mathrm{ml})$ and refluxed at $70^{\circ} \mathrm{C}$ for $4 \mathrm{~h}$. The organic phase containing the iron oleate complex was separated, washed three times with distilled water $(30 \mathrm{ml})$ to extract salts, dried using $\mathrm{MgSO}_{4}$, and finally concentrated. The resulting iron oleate complex was a reddish-brown viscous solid and was stored at $4{ }^{\circ} \mathrm{C}$. A combination of $\mathrm{Fe}(\text { oleate })_{3}\left(2 \mathrm{~g}, 2.2 \times 10^{-3} \mathrm{~mol}\right)$, oleic acid $\left(1.24 \mathrm{~g}, 3.3 \times 10^{-3}\right.$ mol) and octyl ether $(20 \mathrm{~mL})$ was stirred for $1 \mathrm{~h}$ to dissolve the reactants at $100^{\circ} \mathrm{C}$. The temperature was carefully raised to reflux with a heating rate of $5^{\circ} \mathrm{C} / \mathrm{min}$ without stirring for 120 min under air at $288^{\circ} \mathrm{C}$. After cooling down to room temperature, the black suspension of nanocrystals was washed 3 times by addition of ethanol and centrifugation (8000 rpm, $10 \mathrm{~min}$ ). The obtained nanocrystals could be easily suspended in various organic solvents to raise a highly stable suspension which can be stored for several months. The size monodispersity of NPs was improved by applying a size selection precipitation process [17]. The nanoparticles were suspended in hexane at a concentration of $1 \mathrm{mg} / \mathrm{mL}$ and precipitated by adding the same volume of acetone followed by centrifugation. The precipitate was redispersed in THF to prepare a highly stable suspension of coated nanoparticles with a specific concentration of $3.7 \mathrm{mg} \cdot \mathrm{mL}^{-1}$.

Preparation of self-assembled monolayers (SAMs). Freshly cleaned and activated silicon wafers were used for all the preparations. SAMs were prepared by sol-gel chemistry which consists in the hydrolysis of the triethoxysilyl groups and their condensation with hydroxyl groups at the surface of the activated silicon wafer. A solution of the alkoxysilane $(0.01 \mathrm{M})$ was prepared in dry toluene. The silicon wafers were immersed in the solution for 3 hours at $110^{\circ} \mathrm{C}$ under argon. After cooling down to room temperature, the substrates were extensively washed with toluene and dried under a $\mathrm{N}_{2}$ stream.

Characterization of NPs. The NPs were characterized by X-ray diffraction (XRD) using a Bruker D8 Advance equipped with a monochromatic copper radiation, $(\mathrm{K} \alpha=$ $0.154056 \mathrm{~nm}$ ) and a Sol-X detector in the $27-65^{\circ} 2 \theta$ range with a scan step of $0.03^{\circ}$. TEM images were recorded with a TOPCON model 002B transmission electron microscope, operating at $200 \mathrm{kV}$, with a point-to-point resolution of 0.18 $\mathrm{nm}$. The polydispersity in size was calculated from the size measurements of more than 300 nanoparticles. Dynamic light scattering (DLS) measurements were performed on the suspension of nanoparticles in THF using a nano-size MALVERN (nano ZS) apparatus. FTIR spectroscopy was performed using Digilab Excalibur 3000 spectrophotometer (CsI beamsplitter) in the energy range $4000-400 \mathrm{~cm}^{-1}$. The samples were analyzed in the solid state by using an ATR accessory (Single Reflexion Attenuated Total Reflectance).

Assembling of iron oxide nanoparticles on SAMs. SAMs were immersed directly after preparation in the suspension of oleate coated nanoparticles in THF at room temperature for 10 or 90 minutes. The substrates were then placed in an ultrasonic bath in THF to remove any physisorbed nanoparticles and finally dried under a stream of nitrogen.

Characterization of SAMs. The surface activity of mixed SAMs was qualitatively studied by contact angle measurements with water. This technique is very convenient because of its high sensitivity to details of the interfacial structure at the molecular level. Contact angle values were measured with a Digidrop ${ }^{\circledR}$ contact angle goniometer (GBX, France).

Characterization of NPs assemblies on SAMs. Scanning electronic microscopy (SEM) was performed using a JEOL 6700 microscope equipped with a field emission gun (SEMFEG) operating at an accelerating voltage of $3 \mathrm{kV}$. Ellipsometry measurements were performed with a plasmon SD 2300 ellipsometer operating at the single wavelength of $632.8 \mathrm{~nm}$ and a constant angle of $70^{\circ}$. Atomic Force Microscopy (AFM) was performed using a Digital Instrument 3100 microscope coupled with a Nanoscope IIIa recorder. The measurements were done in the tapping mode onto substrates before and after exposition to the nanoparticles suspension. The collected datas were analyzed with the Nanotec WSXM software. ${ }^{53}$

Magnetic characterization of NPs assemblies. The magnetic properties of assemblies were investigated by using a superconducting quantum interference device (SQUID) magnetometer (Quantum Design model MPMS-XL). Magnetizations versus applied magnetic field curves were recorded at $300 \mathrm{~K}$ and $5 \mathrm{~K}$. Zero field cooling (ZFC) and field cooling (FC) curves were recorded from 5 to $300 \mathrm{~K}$ under exposition of an applied magnetic field of $75 \mathrm{G}$. ZFC curves were first recorded from $5 \mathrm{~K}$ to $300 \mathrm{~K}$ after the temperature had been decreased without applying any magnetic field. Then, the FC curves were recorded after the temperature was 
set down again to $5 \mathrm{~K}$ under $75 \mathrm{G}$. The substrates containing the assemblies of nanoparticles were placed in a parallel direction to that of the applied magnetic field. ZFC and FC curves were normalized from the maximum values of the magnetization at the blocking temperature $\left(\mathrm{T}_{\mathrm{B}}\right)$.

\section{RESULTS AND DISCUSSION}

The assembling of nanoparticles following a bottom up approach consists in using nanoparticles as nanobuilding blocks. Therefore, it is of first importance to consider the assembling of independent nanoparticles. Recently, the thermal decomposition method was reported to be very efficient to prepare iron oxide nanoparticles with high stability in solution [15]. This method also enables the high control on the size distribution and morphology such as spheric- [16] and cubic-shaped [18] nanoparticles which directly influence the magnetic properties. Iron oxide nanoparticles were synthesized by the thermal decomposition of an iron oleate precursor at $310^{\circ} \mathrm{C}$ in a high boiling point solvent such as octylether. At the end of the reaction it is very important to wash out the sample by centrifugation and dialysis to remove the side-products of the reaction as well as the excess of surfactant molecules which influence the kinetic of the assembling on SAMs [10]. A size selection precipitation (SSP) procedure was also applied to narrow the size distribution of nanoparticles. Transmission electron microscopy (TEM) shows the high control on the spherical morphology of nanoparticles (Fig. 1a). The size distribution was measured over more than $300 \mathrm{NPs}$ and is centred on $12 \pm 1 \mathrm{~nm}$ with narrow size dispersion (Fig. 1b). The stability of the nanoparticles in THF was investigated by granulometric measurements using dynamic light scattering (DLS) (Fig. 1b) and showed a narrow distribution of the hydrodynamic diameter of $15.9 \pm 1 \mathrm{~nm}$. This value is slightly higher than the size of the nanoparticles since the hydrodynamic diameter includes the oleic acid molecules adsorbed at the NP surface. This result demonstrates the high stability of the suspension and the non aggregation of nanoparticles thanks to the oleic acid which is adsorbed at the NP surface. The composition of the nanoparticles was studied by FTIR spectroscopy. Fig. (1c) shows characteristic bands of iron oxide $\left(\mathrm{vFe}-\mathrm{O}=592 \mathrm{~cm}^{-1}\right)$ and of oleic acid $\left(v \mathrm{C}=\mathrm{O}\right.$ at $1705 \mathrm{~cm}^{-1}$ and $v \mathrm{C}-\mathrm{H}$ at $\left.2840-2930 \mathrm{~cm}^{-1}\right)$.

The structure of the nanoparticles was also investigated by electronic diffraction (Fig. 2a) which shows several intense rings which are all indexed to the $h k l$ plans (220), (311), (400), (422), (511) et (440) of the spinel structure of iron oxide nanoparticles. This is confirmed by X-Ray Diffraction (XRD) measurement which exhibits Bragg peaks indexed for the same spinel structure (Fig. 2b). The comparison with the JCPDS card of magnetite $\left(\mathrm{Fe}_{3} \mathrm{O}_{4}\right.$, file $\left.\mathrm{n}^{\circ} 19-629\right)$ and maghemite $\left(\gamma-\mathrm{Fe}_{2} \mathrm{O}_{3}\right.$, file $\left.\mathrm{n}^{\circ} 39-1346\right)$ shows that the structure is intermediate. These observations correspond to the partial oxidation of the iron (II) at the surface of NPs as reported previously [19].

The preparation of SAMs (Scheme 1) was performed by the reaction of trialkoxysilylated molecules with the silanol groups at the surface of silicon wafers which have been cleaned and activated by an $\mathrm{O}_{2} / \mathrm{Ar}$ plasma. Silicon wafers were immersed for 12 hours at room temperature under inert

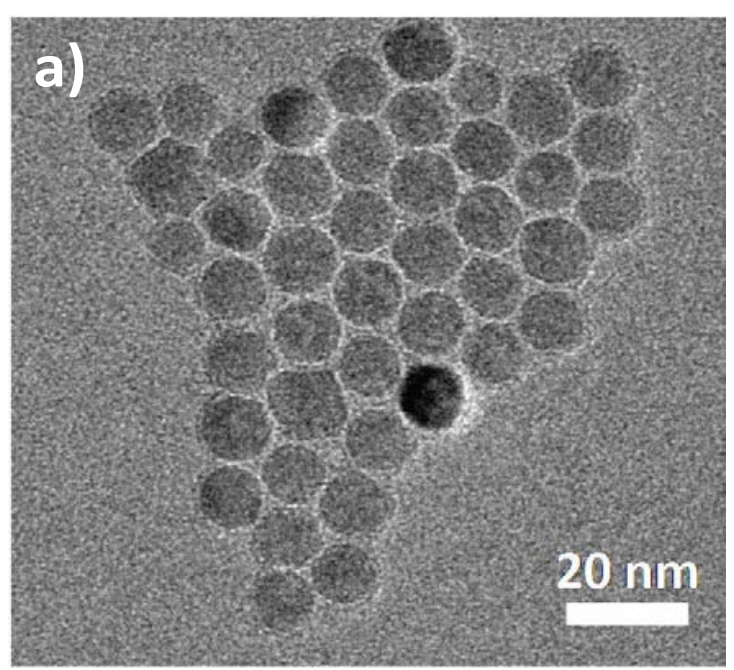

b)
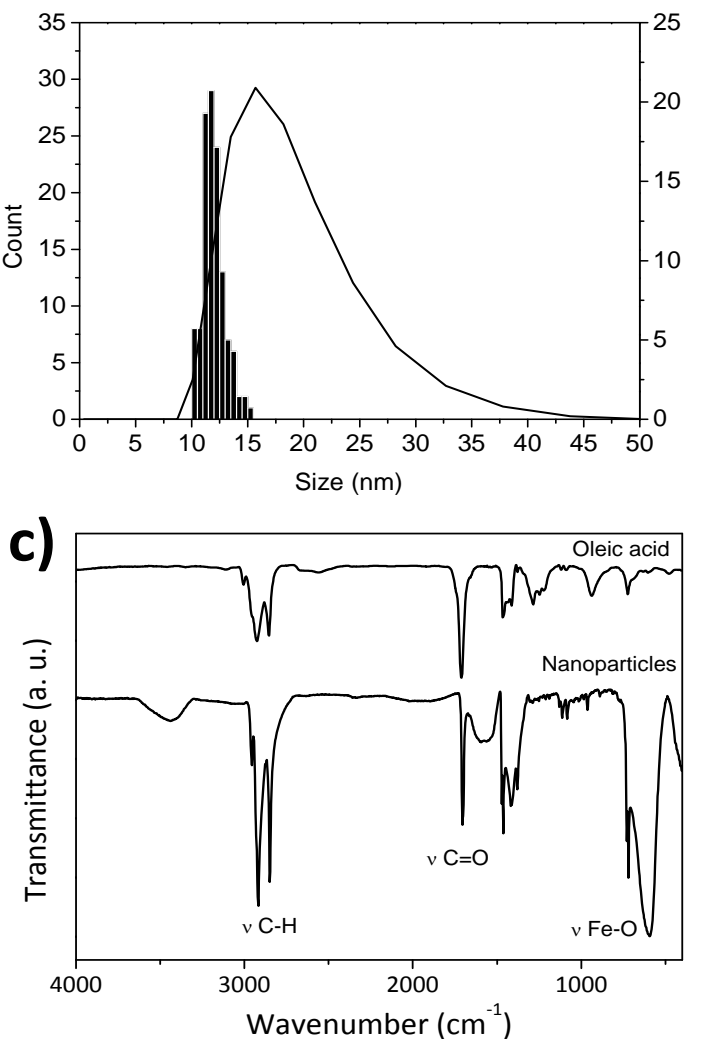

Fig. (1). Iron oxide magnetic nanoparticles coated with oleic acid. a) TEM micrograph. b) Size distribution (histogram) measured from TEM micrographs and hydrodynamic diameter (curve). c) FTIR spectra of oleic acid (top) and of nanoparticles coated with oleic acid (bottom).

atmosphere in a solution of the silylated precursor in dry toluene. After the reaction, the samples were extensively washed with toluene and dried under a nitrogen stream. Two different trialkoxy-silanes were used, the commercial 3aminopropyltrieth-oxysilane (APTES) and the $\mathrm{N}-1$ (triethoxysilylundecyl) thymine (THY-Si) [20], to prepare SAM-NH2 and SAM-THY, respectively (Scheme 1). The 
samples were very easily characterized by contact angle measurements. SAM-NH2 and SAM-THY display contact angles of $78^{\circ}$ and $68^{\circ}$, respectively which correspond to moderately hydrophilic surfaces. These values are higher than that of a freshly cleaned silicon wafer $\left(16^{\circ}\right)$. Nevertheless they agree with SAMs decorated with polar groups at their surface and that present some disorder [21, 22]. The value of SAM-THY is slightly lower than that of SAM-NH2 and can be attributed to the longer alkylene chain of the former which favors a better assembling through Van der Waals interactions compared with the shorter propylene chain of APTES. The higher polarity of the thymine compared with the amine group may also account for that. The thickness of the grafted layer of organotrialkoxysilanes was also measured by ellipsometry. SAM-NH2 and SAMTHY display values of $0.4 \pm 0.1 \mathrm{~nm}$ and $1.2 \pm 0.4 \mathrm{~nm}$, respectively. The measurement was performed at the scale of the whole sample and shows the homogeneity of both SAMs. The difference between these values is in agreement with the different length of the alkylene chains of these two molecules.

The assembling of nanoparticles was performed by immersing SAMs-NH2 and SAM-THY separately in the NPs suspension in THF at room temperature for 10 and 90 min. The SAMs were subsequently removed and extensively washed with THF. They were also exposed to ultrasonication for $10 \mathrm{sec}$ to remove physisorbed NPs on the first layer of nanoparticles. SAMs were finally dried under a nitrogen stream. Scanning electron microscopy (SEM) images show that nanoparticles were deposited on all four SAMs (Fig. 3). Almost no aggregates are observed which confirms the high stability of the suspension and the non aggregation of NPs. For the longer time exposure $(90 \mathrm{~min})$ both $\mathrm{SAM}-\mathrm{NH} 2$ and SAM-THY are covered homogeneously by NPs with similar density (about $70 \%$ of the surface is covered). In contrast, for the shorter time exposure $(10 \mathrm{~min})$ SAM-NH2 shows a lower coverage by NPs $(55 \%)$ than SAM-THY (65\%).

Both SAMs exhibit functional groups at the surface which have the ability to immobilize NPs. The amine group has coordinating abilities while the thymine group induces stronger chelating interactions. The assembling of NPs occurs spontaneously by the reduction of the interfacial energies on specific areas patterned by amine or thymine groups [23]. NPs are coated with oleic acid and exhibit a
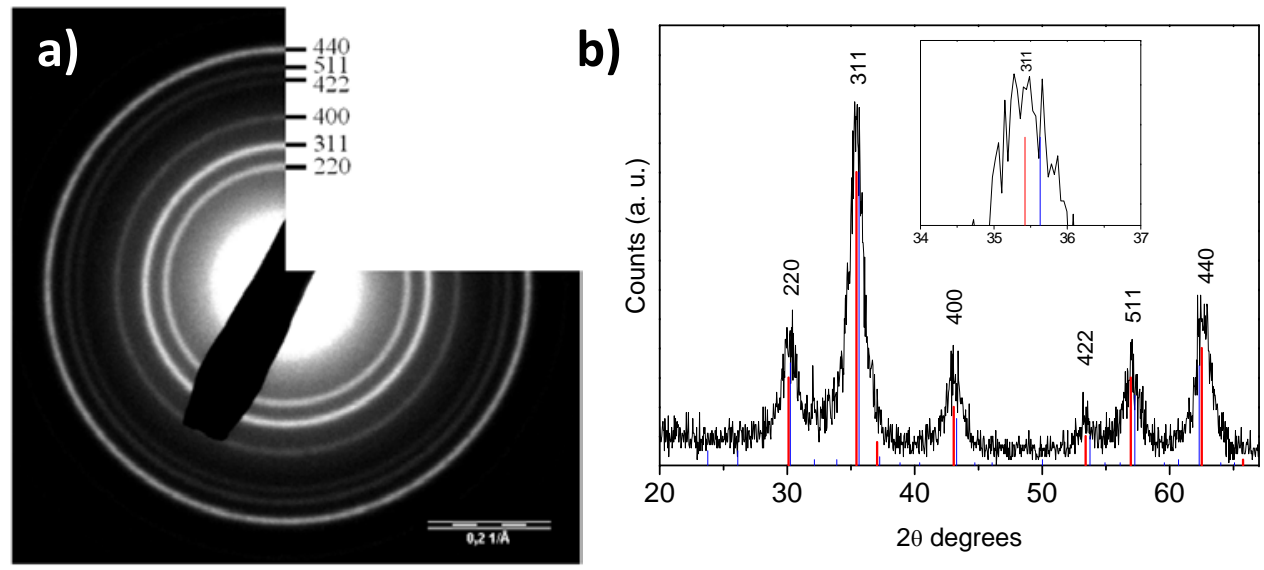

Fig. (2). Structural data of the nanoparticles. a) Electron diffraction pattern. b) X-ray diffraction pattern.
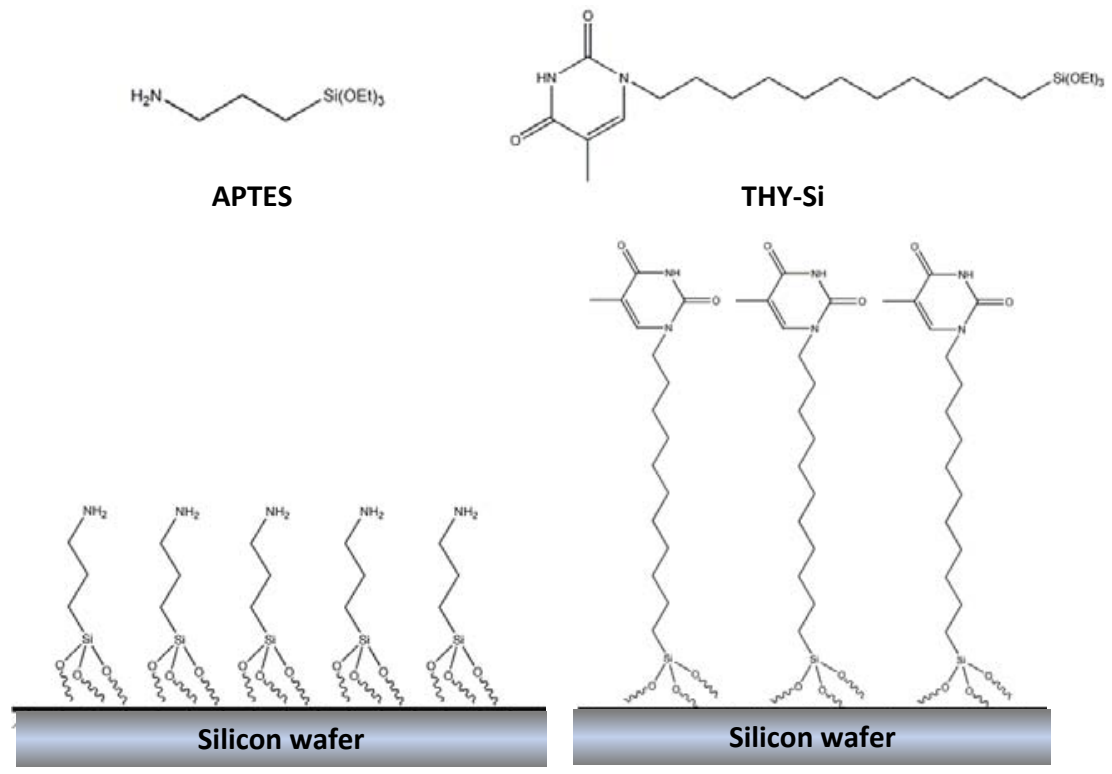

Scheme 1. Organotrialkoxysilane molecules and their corresponding SAMs. 

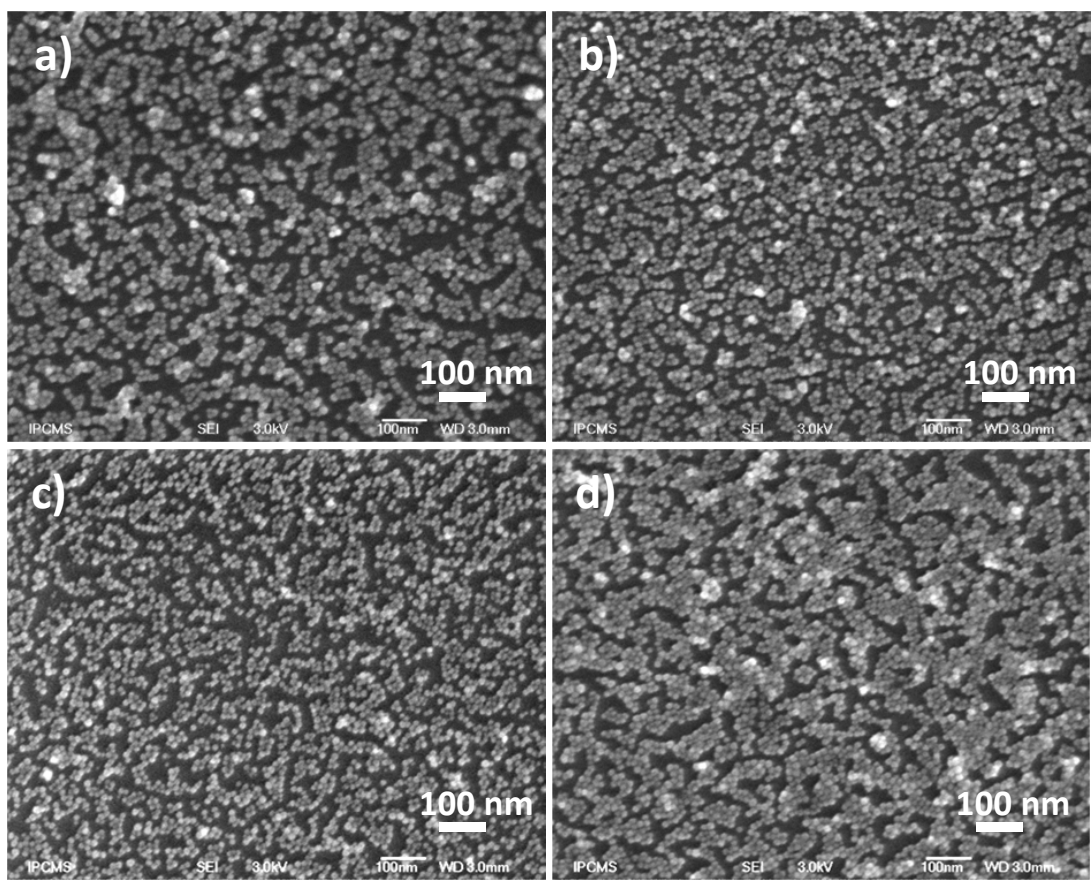

Fig. (3). SEM images of a), b) SAM-NH2 and c), d) SAM-THY immersed in the NPs suspension for a), c) $10 \mathrm{~min}$ and b), d) $90 \mathrm{~min}$.

hydrophobic surface which should avoid their immobilization through interactions with polar terminal groups from the SAM surface. Indeed, the immobilization of NPs on the SAMs requires the NPs surface to be directly available to interact with amine or thymine groups at the surface of the SAMs. However, oleic acid molecules interact at the NPs surface by weak interactions. Therefore, there is equilibrium between molecules at the NPs surface and free molecules in solution. This equilibrium depends on the $\mathrm{NPs} /$ oleic acid molecules ratio as well as the solvent. The high density of coordinating or chelating groups at the SAM surface also significantly modifies the equilibrium [10]. Here, the reaction condition favors the free molecules in solution and therefore the NPs surface is partially uncovered and amine or thymine groups interact directly with NPs. Nevertheless, the kinetics of the assembling of NPs are clearly influenced by the chemical nature of the functional groups at the surface of the SAMs. Coordinating amine groups induce weaker interactions than chelating thymine groups. Therefore, as we observed previously with hydroxyl and carboxylic acid terminal groups at the SAM surface, the density of assembled NPs is modified by the kinetics of the reaction [10]. Moreover, the immobilization of NPs from the solution to the substrate occurs randomly in domains. These domains extend with time and may be favored by dipolar interaction between NPs. It shows that the immobilization of NPs on the SAM may initially happen randomly but it is further directed by dipolar interactions between NPs. Consequently, this partially random mechanism results in the partial covering of the surface by NPs which always remain lower than the highest $2 \mathrm{D}$ compacity $(0.9)$ corresponding to the highly ordered hexagonal compact packing $(h c p)$.

The immobilization of NPs was also investigated on a larger scale by ellipsometry. All samples, except SAM-NH2 after $10 \mathrm{~min}$, display an increased thickness of $9 \mathrm{~nm}$ which agrees well with the immobilization of a monolayer of 12 $\mathrm{nm}$-sized NPs. This lower value than the NPs diameter can be related to the incomplete coverage of the surface by NPs and also by the fact that NPs are spherical. Contact angle measurements were also performed after the immobilization of NPs (Table 1). The values are higher than for the corresponding SAM-NH2 and SAM-THY and agree with the immobilization of NPs coated with oleic acid molecules on the side exposed to air and hydrophobic surfaces. Surfaces with the highest density in NPs display the highest values of contact angle. In contrast, the contact angle measured for SAM-NH2 with a shorter exposure time (10 minutes) to the NPs suspension is slightly lower which is in agreement with larger unoccupied areas of the SAM.

Table 1. Contact Angle Measured on SAMs After Immobilization of Nanoparticles. ${ }^{a}$ Time of Immersion in the NPs Suspension

\begin{tabular}{|c|c|c|}
\hline & Time $^{\text {a }}$ (min) & Contact Angle \\
\hline \hline SAM-NH2 & 10 & 93 \\
\hline SAM-NH2 & 90 & 107 \\
\hline SAM-THY & 10 & 105 \\
\hline SAM-THY & 90 & 108 \\
\hline
\end{tabular}

The topography of the SAM-THY was also studied by Atomic Force Microscopy (AFM) in tapping mode after immersion in the NPs suspension for $10 \mathrm{~min}$. The analysis was performed on several regions to characterize the SAMs before and after deposition of NPs (Fig. 4). After immersion in the NPs suspension, the SAM-THY is featured by a higher roughness $(2.2 \pm 0.1 \mathrm{~nm})$ than the corresponding SAMs before immersion $(0.5 \pm 0.1 \mathrm{~nm})$. These results are very similar to those obtained from SAMs prepared on gold substrates $[10,11]$. It appears that the higher roughness of gold substrates compared with silicon wafer does not influence the assembling of NPs. Topographic height 

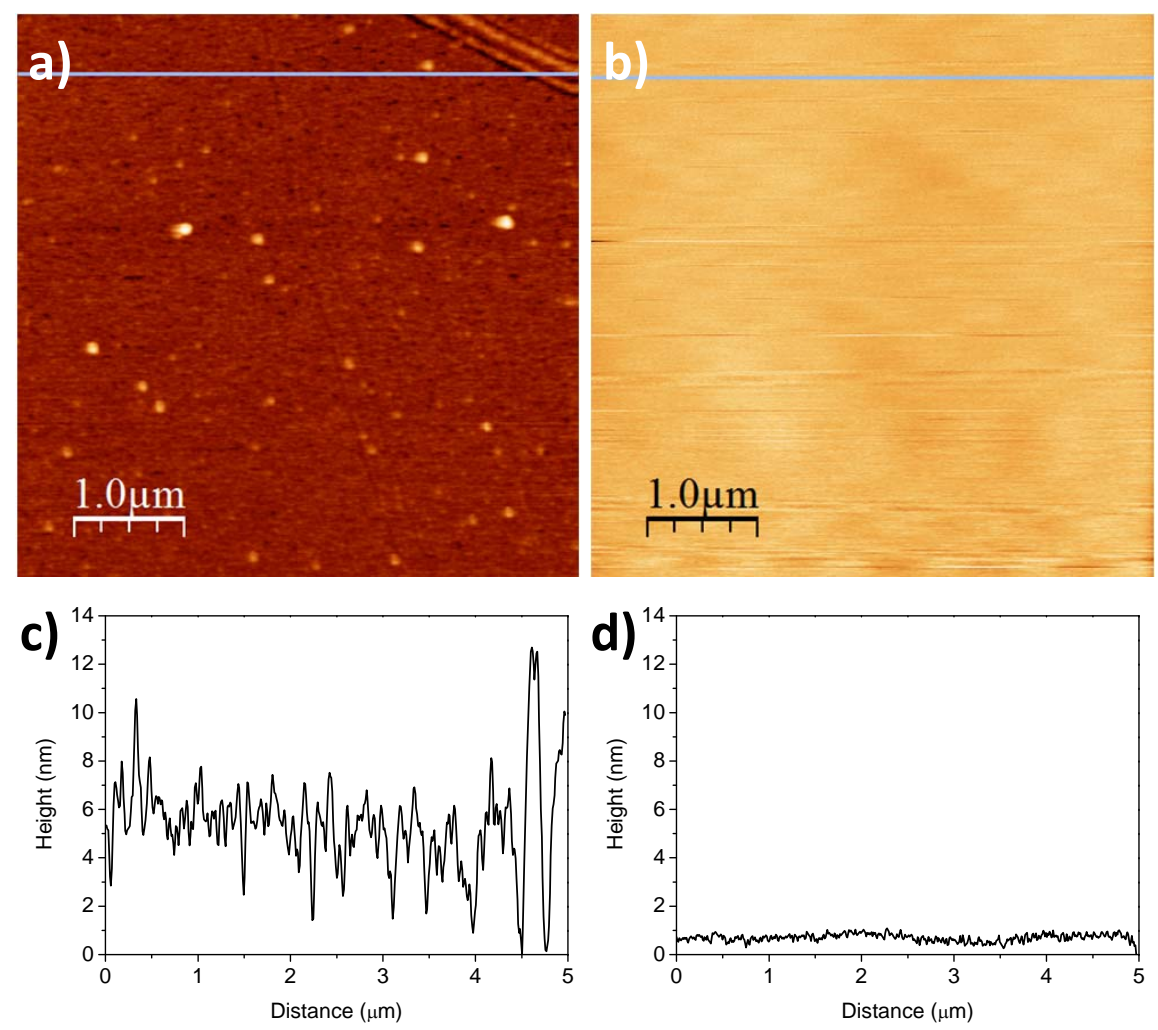

Fig. (4). AFM images and cross section profile corresponding to the line on the upper part of the AFM images. a) and c) SAM-THY 10 minutes after immersion in the NP suspension. b) and d) SAM-THY before immersion.

measurements demonstrate the homogeneous assembling of NPs on the SAM with a high coverage density. Some unoccupied areas are observed as the occurrence of darker regions but do not exceed $15 \%$. Such free areas enable us to confirm the formation of a monolayer of nanoparticles as revealed by the surface cross section which shows average height differences of about $8 \mathrm{~nm}$ which is consistent with the particle size. Individual nanoparticles are not clearly observed and appear as irregularly shaped blocks because of their coating with oleic acid and of usual tip effects upon scanning. In addition, SAMs of organoalkoxysilanes exhibit a very low roughness and a very flat surface cross section similarly to those of silicon wafer (not shown). This agrees with the self-assembling of molecules as a continuous layer with an homogeneous thickness.

The magnetic properties of SAM-THY and SAM-NH2 after immersion in the NPs suspension for 10 min have been studied using a Scanning Quantum Interference Device (SQUID) magnetometer. Magnetization curves were recorded as a function of the temperature from $5 \mathrm{~K}$ to $300 \mathrm{~K}$ without an applied magnetic field (zero field cooled curve, ZFC) and under a magnetic field of $75 \mathrm{G}$ (field cooled curve, FC) (Fig. 5a). Both curves are highly characteristic of 12 $\mathrm{nm}$-sized nanoparticles which are superparamagnetic at room temperature. The maximum of the ZFC is attributed to the blocking temperature $\left(T_{B}\right)$, i.e. the transition between the superparamagnetic and the ferromagnetic state. Below $T_{B}$, the magnetic anisotropy of the NPs becomes stronger than
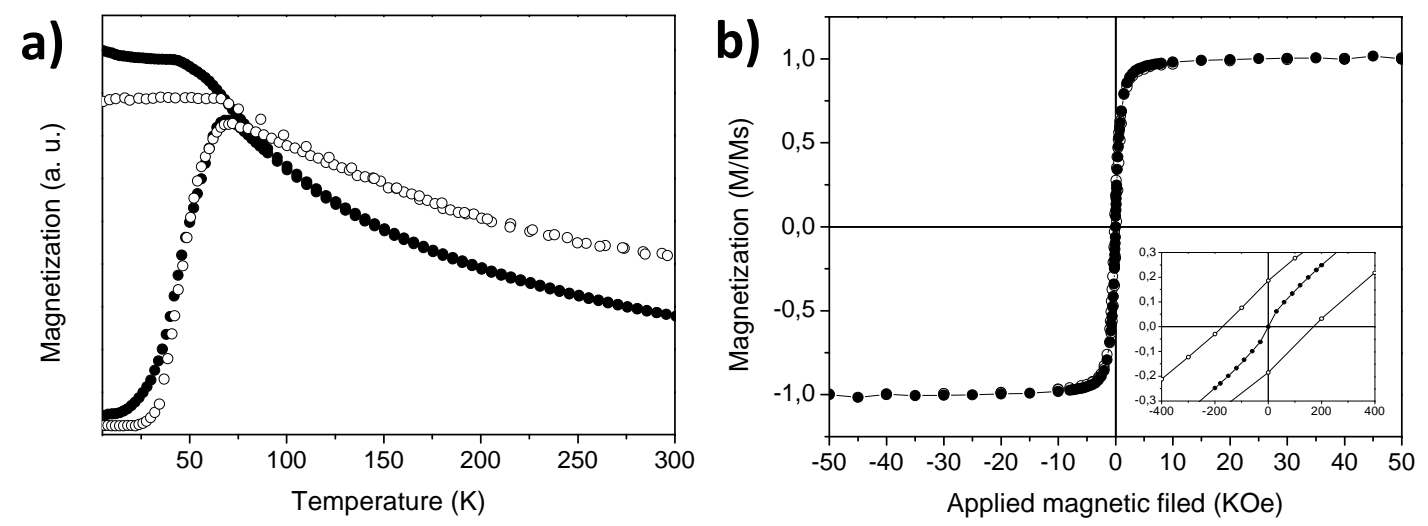

Fig. (5). Magnetic measurements on SAM-NH2 (closed symbol) and SAM-THY (opened symbol) after immersion in the NPs suspension for 10 min. a) Zero field cooled (ZFC) and field cooled (FC) curves. b) Magnetization versus an applied field at $300 \mathrm{~K}$ and $5 \mathrm{~K}$ of SAM-THY after exposure to the NPs suspension. 
the thermal agitation energy. Both samples are featured by similar values of $69 \mathrm{~K}$ and $71 \mathrm{~K}$, respectively for SAM-NH2 and SAM-THY. These results correspond to dipolar interactions with similar strength because both films have similar structure. The only difference between ZFC consists in the broadening of the ZFC related to SAM-THY which demonstrates a larger distribution of $\mathrm{T}_{\mathrm{B}}$. This phenomenon may result from the higher NPs density on SAM-THY than on SAM-NH2. Indeed, the NPs being assembled in a random fashion, the dipolar interactions are more complex as a result of the diminution of the interparticle distance. According to FCs, the saturation in magnetization below $T_{B}$ corresponds to the fact that all NPs interact through dipolar interactions in the film. Magnetization curves were also recorded as a function of the applied field and are very similar for both samples. Fig. (5b) shows $\mathrm{M}(\mathrm{H})$ curves at $300 \mathrm{~K}$ and $5 \mathrm{~K}$ for SAM-THY after immersion in the NPs suspension for 10 min. At $300 \mathrm{~K}$, no hysteresis is observed whereas at $5 \mathrm{~K}$, a coercive field of about $170 \mathrm{Oe}$ is measured. These curves are characteristic of superparamagnetic and ferromagnetic states, respectively. NPs assembled in the same conditions on SAM-NH2 show very similar curves which is indicative similar magnetic properties.

\section{CONCLUSION}

Magnetic iron oxide nanoparticles have been assembled in 2D monolayers by using SAMs of organotrialkoxysilanes. The properties of NPs such as their non agglomeration and their high stability in suspension are highly important to consider their precise assembling following a bottom-up approach. The chemical nature of the functional head groups at the SAM surface clearly influences the kinetic of the assembling process. Thymine groups with strong chelating abilities result in faster kinetics of the NPs immobilization than amine groups. Finally, the surface density of NPs slightly influences the magnetic properties of the film, and more precisely the strength of the dipolar interactions between nanoparticles.

\section{ACKNOWLEDGEMENT}

The authors thank the Agence Nationale de la Recherche (ANR), Programme Blanc, project $n^{\circ}$ NT09_459731 entitled "MAGARRAY".

\section{CONFLICT OF INTEREST}

The authors confirm that this article content has no conflicts of interest.

\section{REFERENCES}

[1] Zeng H, Black CT, Sandstrom RL, Rice PM, Murray CB, Sun S. Magnetotransport of magnetite nanoparticle arrays. Phys Rev B 2006; 73(2): 020402

[2] Reasmey PT, Carrey J, Desvaux C, Grisolia J, Renaud P, Chaudret B. Transport in superlattices of magnetic nanoparticles: coulomb blockade, hysteresis, and switching induced by a magnetic field. Phys Rev Lett 2007; 99(17): 176805.
[3] Tan RP, Carrey J, Respaud M, Desvaux C, Renaud P, Chaudret B. $3000 \%$ high-field magnetoresistance in super-lattices of $\mathrm{CoFe}$ nanoparticles. J Magn Magn Mater 2008; 320(6): L55.

[4] Kinge S, Crego-Calama M, Reinhoudt DN. Self-assembling nanoparticles at surfaces and interfaces. Chem Phys Chem 2008; 9 (1): $20-42$

[5] Grancharov SG, Zeng H, Sun S, et al. A bio-functionalization of monodisperse magnetic nanoparticles and their use as biomolecular labels in a magnetic tunnel junction based sensor. J Phys Chem B 2005; 109(26): 13030 .

[6] Bae SS, Lim DK, Park JI, Kim S, Cheon J, Lee WR. Selectively assembled co nanoparticle stripes prepared by covalent linkage and microcontact printing. J Phys Chem B 2004; 108(8): 2575-9.

[7] Puntes VF, Gorostiza P, Aruguete DM, Bastus NG, Alivisatos AP. Collective behaviour in two-dimensional cobalt nanoparticle assemblies observed by magnetic force microscopy. Nat Mater 2004; 3(4): 263.

[8] Alexander Z, Gregory L, Sidney RC, Ron N. Adsorption-induced magnetization of pbs self-assembled nanoparticles on GaAs. Adv Mater 2008; 20(13): 2552-5.

[9] Zakrassov A, Bitler A, Etgar L, Leitus G, Lifshitz E, Naaman R. Controlling the anisotropic magnetic dipolar interactions of PbSe selfassembled nanoparticles on GaAs. Phys Chem Chem Phys 2009; 11(35): 7549-52.

[10] Pichon BP, Demortiere A, Pauly M, Mougin K, Derory A, Begin-Colin S. 2D assembling of magnetic iron oxide nanoparticles promoted by SAMs used as well-addressed surfaces. J Phys Chem C 2010; 114(19): 9041.

[11] Pichon BP, Pauly M, Marie P, Leuvrey C, Begin-Colin S. Tunable magnetic properties of nanoparticles $2 \mathrm{D}$ assemblies addressed by mixed SAMs. Langmuir 2011; 27: 6235-43.

[12] Tamada K, Hara M, Sasabe H, Knoll W. Surface phase behavior of nalkanethiol self-assembled monolayers adsorbed on au(111): an atomic force microscope study. Langmuir 1997; 13(6): 1558.

[13] Choi I, Kim Y, Kang S K, Lee J, Yi J. Phase separation of a mixed selfassembled monolayer prepared via a stepwise method. Langmuir 2006; 22(11): 4885-9.

[14] Moreau JJE, Pichon BP, Arrachart G, Man MWC, Bied C. Nanostructuring organo-silicas: combination of intermolecular interactions and molecular recognition properties to generate selfassembled hybrids with phenylene or adenine center dot center dot center dot thymine bridging units. New J Chem 2005; 29(5): 653-8.

[15] Park J, An K, Hwang Y, et al. Ultra-large-scale syntheses of monodisperse nanocrystals. Nat Mater 2004; 3(12): 891.

[16] Demortiere A, Panissod P, Pichon BP, et al. Size-dependent properties of magnetic iron oxide nanocrystals. Nanoscale 2011;3(1): 225.

[17] Pauly M, Pichon BP, Demortière, et al. Large 2D monolayer assemblies of iron oxide nanocrystals by the Langmuir-Blodgett technique. Superlatt Microstruct 2009; 46(1-2): 195.

[18] Pichon BP, Gerber O, Lefevre C, et al. Microstructural and magnetic investigations of wüstite-spinel core-shell cubic-shaped nanoparticles. Chem Mater 2011; 23: 2886-900.

[19] Daou TJ, Pourroy G, Begin-Colin S, et al. Hydrothermal synthesis of monodisperse magnetite nanoparticles. Chem Mater 2006; 18(18): 4399-404.

[20] Moreau JJE, Pichon BP, Arrachart G, Man WC, Bied C. Nanostructuring organo-silicas: combination of intermolecular interactions and molecular recognition properties to generate selfassembled hybrids with phenylene or adeninethymine bridging units. New J Chem 2005; 29(5): 653.

[21] Arima Y, Iwata H. Effect of wettability and surface functional groups on protein adsorption and cell adhesion using well-defined mixed selfassembled monolayers. Biomaterials 2007; 28(20): 3074.

[22] Faucheux N, Schweiss R, Lützow K, Werner C, Groth T. Selfassembled monolayers with different terminating groups as model substrates for cell adhesion studies. Biomaterials 2004; 25 (14): 2721.

[23] Lin Y, Boker A, Skaff, et al. Nanoparticle assembly at fluid interfaces: structure and dynamics. Langmuir 2004; 21(1): 191-4. 